\title{
ORIGINAL
}

\section{ESTUDIO CASOS-CONTROL DE MARCADORES DE ESTRÉS OXIDATIVO Y METABOLISMO DEL HIERRO PLASMÁTICO EN LA ENFERMEDAD DE PARKINSON (*)}

Rosa Larumbe Ilundáin (1), Jose Vicente Ferrer Valls (1), José Javier Viñes Rueda (2), David Guerrero (1), Pablo Fraile (3).

(1) Centro de Investigación Biomédica. Servicio Navarro de Salud.

(2) Servicio de Docencia e Investigación. Departamento de Salud. Gobierno de Navarra.

(3) Laboratorio del Instituto de Salud Pública. Servicio Navarro de Salude.

(*) Trabajo financiado por el Departamento de Salud, Gobierno de Navarra. (Proyecto n. ${ }^{\circ}$ 1233/94)

\section{RESUMEN}

Fundamento: Existe cada vez más evidencia de la implicación de mecanismos de estrés oxidativo en la enfermedad de Parkinson. Se han descrito en la sustancia negra niveles menores de GSH, aumento del depósito de hierro, aumento de los productos derivados de la peroxidación lipídica y alteraciones del complejo I mitocondrial. Sin embargo, son escasos los estudios de niveles de antioxidantes en sangre periférica y de la influencia del consumo de nutrientes en el desarrollo de la enfermedad.

Métodos: Se estudia en un grupo de 79 pacientes con enfermedad de Parkinson idiopática y en un grupo control de 107 sujetos, equiparados por edad, sexo y lugar de residencia, los niveles plasmáticos de: Glutatión reducido (GSH), Malonildialdehído (MDA), ácido úrico, tocoferol, -caroteno, licopeno y diversos parámetros del metabolismo del hierro. Así mismo, se estima el consumo de ciertos antioxidantes a partir de una encuesta dietética.

Resultados: Hemos encontrado diferencias significativas ( $p$ 0,001) en los niveles plasmáticos de GSH entre casos $(0,10$ $\mathrm{mol} / \mathrm{ml} \quad 0,06)$ y controles $(0,29 \mathrm{~mol} / \mathrm{ml} \quad 0,12)$. De igual modo, los niveles de ácido úrico en plasma fueron más bajos (p 0,05$)$ en los casos $(4,96 \mathrm{mg} / \mathrm{ml} \quad 1,96)$ que en los controles $(5,39 \mathrm{mg} / \mathrm{ml} \quad 1,13)$. No hemos encontrado diferencias significativas de los niveles plasmáticos de MDA, tocoferol, -caroteno y licopeno. Respecto al metabolismo del hierro, en los pacientes con EP encontramos valores de ferritina y de transferrina significativamente mayores que en los controles, con un índice de saturación de la transferrina menor (p 0,05). El hierro no mostró cambios significativos entre casos y controles.

Conclusiones: Los resultados de este estudio apoyan la posible implicación del estrés oxidativo en la patogénesis de la enfermedad de Parkinson y, a la vez, evidencian alteraciones de algunos parámetros en sangre periférica en concordancia con hallazgos conocidos en la sustancia negra.

Palabras clave: Ácido úrico. Enfermedad de Parkinson. Estrés oxidativo. Glutatión. Hierro. Tocoferol. Transferrina.

Correspondencia:

Rosa Larumbe

Centro de Investigación Biomédica. Servicio Navarro de Salud

Recinto Hospital de Navarra.

31008. Pamplona.

Navarra
ABSTRACT

\section{Case-control Study of Oxidative Stress and Plasma Iron Metabolism Markers in Parkinson's Disease}

Background: Increasingly growing evidence exists of the involvement of oxidative stress mechanisms in Parkinson's disease. Lower levels of GSH in the sustantia nigra, an increase in iron buildup, an increase in the byproducts of lipid peroxidation and alterations in the mitochondrial complex I have been described. However, few studies have been made of levels of antioxidants in the peripheral bloodstream and of the influence of the intake of nutrients on the development of this disease.

Methods: In a group of 79 patients afflicted with idiopathic Parkinson's disease and a control group comprised of 107 subjects, compared by age, sex and place of residence, the lowered levels in the plasma of glutathione (GSH), malon dialdehyde (MDA), uric acid, tocopherol, -carotene, lycopene and different iron metabolism parameters were studied. Likewise, the intake of certain antioxidants was estimated based on a dietary survey.

Results: Significant differences (p 0.001) were found in the plasma levels of GSH between cases $\left(\begin{array}{lll}0.10 & \mathrm{~mol} / \mathrm{ml} & 0.06\end{array}\right)$ and controls $\left(\begin{array}{lll}0.29 & \mathrm{~mol} / \mathrm{ml} & 0.12\end{array}\right)$. Likewise, the plasma levels of uric acid were lower (p 0.05) in the cases $(4.96 \mathrm{mg} / \mathrm{ml}$ 1.96) than in the control groups $(5.39 \mathrm{mg} / \mathrm{ml} \quad 1.13)$. No significant difference was found in the plasma levels of MDA, tocopherol, -carotene and lycopene. With regard to iron metabolism, significantly higher ferritine and transferrin values were found in the patients with EP than in the control group, showing a lower transferrin saturation percentage ( $\mathrm{p}$ 0.05). The iron showed no significant changes between cases and control groups.

Conclusions: The results of this study support the possible involvement of oxidative stress in the pathogenesis of Parkinson's disease and reveal, in turn, alterations in some peripheral blood parameters in keeping with known findings in the sustantia nigra.

Keywords: Uric acid. Parkinson's disease. Oxidative stress. Glutathione. Iron. Tocopherol. Transferrin. 


\section{INTRODUCCIÓN}

Aunque todavía no se conoce la causa que provoca la muerte neuronal en la enfermedad de Parkinson (EP), parece probable la implicación de procesos de estrés oxidativo en la patogénesis de esta enfermedad. Diversos trabajos demuestran la presencia de niveles bajos de glutatión reducido (GSH) en la sustancia negra de cerebros de pacientes con EP, como un hecho temprano en el curso de la enfermedad ${ }^{1,2}$. El GSH es el sistema enzimático antioxidante más importante de la célula, siendo capaz tanto de inactivar radicales libres de oxígeno (RLO) como de reparar moléculas recientemente oxidadas $^{3}$. En concordancia con la disminución de GSH, se ha encontrado un incremento de la peroxidación lipídica, expresado por la elevación de los niveles de malonildialdehído (MDA) en la sustancia negra de pacientes con $\mathrm{EP}^{4}$. También han sido descritas alteraciones en la actividad del complejo I mitocondrial en las neuronas de la sustancia negra ${ }^{5}$, apoyando la implicación de los RLO en la patogénesis de la enfermedad, puesto que es en la mitocondria donde más procesos oxidativos se producen. Trabajos recientes sugieren incluso, que el proceso de envejecimiento celular se inicia en la mitocondria, dependiendo directamente de fenómenos oxidativos ${ }^{6}$. Otras alteraciones metabólicas encontradas en la sustancia negra se centran en el metabolismo del hierro, cuya presencia activa induce procesos oxidativos. Se ha descrito un incremento del contenido total de hierro en la sustancia negra de cerebros de pacientes con EP, así como un aumento de ferritina y moléculas transportadoras ${ }^{7}$.

La mayor parte de trabajos que han investigado las implicaciones del estrés oxidativo en el desarrollo de la EP se han realizado en sustancia negra y en el líquido cefalorraquí$\mathrm{deo}^{8-9}$. Son pocos los estudios realizados en sangre periférica, y con resultados no concluyentes. Se han descrito niveles más bajos de hierro circulante, ferritina y transferrina en pacientes con EP que en sujetos contro- les ${ }^{10,11}$. El estudio de los niveles de diversos aminoácidos, bilirrubina, -tocoferol, vitamina $\mathrm{C}$, -carotenos, fosfolipasa A2, catalasa y MDA, no ha aportado hasta el momento datos concluyentes ${ }^{12-16}$. Algunos autores han señalado una reducción en los niveles de ácido úrico en la $\mathrm{EP}^{17}$, lo cual es acorde con la implicación del estrés oxidativo en la enfermedad.

La hipótesis de que la EP pudiera desarrollarse en el contexto de un disbalance entre las sustancias prooxidantes y los mecanismos antioxidantes en la sustancia negra, ya sea como factor fundamental o asociado, ha llevado también a estudiar el papel de la dieta, concretamente los compuestos antioxidantes, en la aparición y desarrollo de la enfermedad. Una de las asociaciones más estudiadas ha sido la ingesta de vitamina $\mathrm{E}$, molécula con importante capacidad antioxidante. Tanto es así, que algunos estudios han sugerido que la vitamina $\mathrm{E}$ pudiera ser un factor protector frente al desarrollo de la $\mathrm{EP}^{18,19}$, aunque otros posteriores no han podido confirmar este extremo ${ }^{20,21}$.

Partiendo de esta situación poco clarificadora, nos decidimos a realizar un estudio en un grupo de pacientes con EP idiopática de reciente diagnóstico, y en un grupo control apareado por edad, sexo y lugar de residencia, analizando el consumo estimado de antioxidantes a través de la historia de dieta, además de diversos parámetros bioquímicos en sangre periférica: sustancias antioxidantes, metabolismo del hierro y productos derivados del daño oxidativo. De acuerdo a la hipótesis planteada, esperaríamos encontrar niveles menores de antioxidantes plasmáticos en los pacientes con EP que en el grupo control.

\section{SUJETOS Y MÉTODOS}

Se incluyó en el estudio a 79 personas con EP idiopática diagnosticadas según los criterios de la Sociedad del Banco de Cerebros del Reino Unido ${ }^{22}$. Los casos proceden del 
registro de casos incidentes en el período 1994-95, realizado en una primera fase del estudio epidemiológico de la EP en la Comunidad Foral de Navarra, dirigido por nosotros mismos. De los 98 pacientes incluidos en el registro inicial, en 82 casos se realizaron determinaciones analíticas y encuesta dietética en una segunda fase del estudio. De los que no se obtuvo muestra sanguínea para esta segunda fase, dos casos habían fallecido entre la fecha de incidencia y la realización de este estudio, tres habían cambiado de domicilio fuera de Navarra, uno desarrolló graves problemas de memoria, otro caso presentó una enfermedad irreversible y nueve casos no desearon participar. Las características clínicas de estos últimos no diferían de las del grupo seleccionado. De los 82 casos a los que se realizó examen analítico, tres se excluyeron por presentar cifras altas de ácido úrico $(>7,5)$. Todos fueron evaluados con la escala unificada de valoración del parkinsonismo $\left(\right.$ UPDRS) ${ }^{23}$ y con la escala de Hoehn y $\mathrm{Yahr}^{24}$. Sesenta y cinco pacientes se encontraban en tratamiento con L-Dopa más inhibidor exclusivamente o en asociación con otros fármacos antiparkinsonianos (bromocriptina, 5; pergolide, 8; deprenilo, 20), mientras que 14 pacientes no estaban tratados con L-Dopa.

Los controles se obtuvieron a través del registro de altas hospitalarias de la misma
Comunidad y Sistema Sanitario al que pertenecen los casos, entre las altas producidas en el mismo período de tiempo ${ }^{25}$. Para cada caso se localizaron cuatro controles del mismo año de nacimiento, sexo y área geográfica de residencia. Todos fueron invitados a participar en el estudio sucesivamente, para garantizar entre uno y dos controles por caso. Se obtuvo la colaboración de 131 personas equiparadas por edad, sexo y lugar de residencia. De ellas se excluyeron 24 por presentar cifras elevadas de ácido úrico, por lo que el grupo control quedó constituido por 107 sujetos.

Los criterios de exclusión en casos y controles fueron: demencia o trastorno cognitivo manifiesto, investigados mediante el Mini Mental State ${ }^{26}$; neoplasias activas; historia de enfermedades sistémicas graves; hiperuricemia (ácido úrico>7,5).

Todos los pacientes y controles incluidos en el estudio, lo hicieron por deseo propio, una vez informados con el detalle necesario de la finalidad del mismo, incluyendo la necesidad de una encuesta nutricional y una extracción de sangre periférica por venopunción simple.

Los datos demográficos y clínicos de todos los sujetos se hallan recogidos en la tabla 1 .

Tabla 1

Datos demográficos y clínicos. Los datos cuantitativos se representan por la media y la SD

\begin{tabular}{|lcc|}
\hline & $\begin{array}{c}\text { Pacientes con EP } \\
n=79\end{array}$ & $\begin{array}{c}\text { Controles } \\
n=107\end{array}$ \\
\hline Edad (años) & $69,79(8,60)$ & $70,29(7,9)$ \\
Hombres & 50 & 66 \\
Mujeres & 29 & 41 \\
MMS & $26,77(2,96)$ & $27,53(3,25)$ \\
Tiempo de evolución de la enfermedad desde el & & \\
diagnóstico (años) & $3,63(2,70)$ & \\
Puntuación en la escala UPDRS & $25,39(11,78)$ & \\
Escala de Hoehn y Yahr & $2,17(0,44)$ & \\
\hline
\end{tabular}

MMS: Mini Mental State

UPDRS: Unified Parkinson's Disease Rating Scale 
Se realizaron los siguientes estudios:

\section{Encuesta EPIC de historia de $\operatorname{dieta}^{27}$}

Se trata de una entrevista estructurada informatizada en la que se recoge la historia de dieta referida al período previo al diagnóstico de la enfermedad, en los casos con EP; y durante el año anterior a la entrevista, en los controles. Se hizo referencia a la dieta previa al diagnóstico, en los casos, por entender que tras establecerse el diagnóstico, se podrían haber cambiado los hábitos alimenticios. Hay que tener en cuenta que se trata de casos de reciente diagnóstico, por lo que el período al que se refiere la encuesta es, en la mayoría, el correspondiente a uno o dos años anteriores y por tanto casi equiparable al período al que se refiere la encuesta de los controles. Se recoge el patrón de ingesta habitual de una semana tipo. A partir de ella se calcula la cantidad de nutrientes y grupos de alimentos consumidos, cuya explotación ha sido autorizada por NDiet Soft SL. A efectos del presente trabajo hemos analizado el consumo de dos vitaminas con conocido poder antioxidante: vitamina $\mathrm{C}$ y vitamina $\mathrm{E}$.

\section{Estudio analítico}

Toma de muestras: Se tomaron muestras de sangre periférica mediante punción venosa, y con los sujetos en ayunas. Se obtuvo sangre completa, suero y plasma pobre en plaquetas. Se realizaron las siguientes determinaciones:

Estatus nutricional. Se midieron proteínas totales, albúmina, triglicéridos, LDL y HDL, además de la hematimetría y ionograma habituales. Todo ello se realizó según la técnica automatizada del laboratorio con LX2O Beckman.

Metabolismo del hierro. Se determinaron los niveles plasmáticos de hierro, ferritina, transferrina y el índice de saturación de la transferrina, según la técnica automatizada del laboratorio con LX2O Beckman.
Estatus antioxidante. Se estudió GSH, -tocoferol, -carotenos y licopeno. El GSH se determinó a partir de sangre total, que fue tratada con ácido perclórico a una concentración final del 6\%, obteniéndose el sobrenadante tras agitar con vortex y centrifugar a $11.340 \mathrm{G}$ durante 10 minutos. Teniendo en consideración la rapidez y facilidad con la que se oxida el GSH, se trató la muestra in situ, inmediatamente después de ser extraída. Se utilizó la técnica enzimática de la glutation transferasa para cuantificar la molécula, leyendo los incrementos de densidad óptica a $340 \mathrm{~nm}^{28}$.

El -tocoferol, -carotenos y licopeno, se determinaron en suero mediante high performance liquid chromatography (HPLC) según describe Thurnham y cols ${ }^{29}$. El plasma, previamente desproteinizado con etanol, y adicionado con acetato de tocoferol, se extrajo con heptano. Se evaporó la fase orgánica, se reconstituyó con fase móvil y se inyectó en el cromatógrafo. La columna fue una Hypersil ODS 5 um (200 46 mm) a un flujo de $0,5 \mathrm{~mL} / \mathrm{min}$. Los picos correspondientes a los analitos fueron identificados y cuantificados mediante detector PDA.

Metabolitos resultantes de lesión celular por RLO. Se midieron los niveles plasmáticos de MDA. Se realizó a partir de plasma pobre en plaquetas, obtenido tras mezclar la sangre con citrato $\mathrm{Na}(0.1 \mathrm{M})$, mediante técnica colorimétrica y cuyos incrementos de densidad óptica se leían a $520 \mathrm{~nm}^{30}$.

\section{RESULTADOS}

Los indicadores bioquímicos del estado nutricional se encuentran recogidos en la tabla 2 . No se han encontrado diferencias significativas, excepto un menor nivel de uratos en los pacientes $(\mathrm{p}<0,05)$.

En la estimación del consumo de diversos nutrientes con poder antioxidante se encontró un mayor consumo de vitamina $\mathrm{C}$ y menor de vitamina $\mathrm{E}$ en los casos (tabla 3). 
ESTUDIO CASO-CONTROL DE MARCADORES DE ESTRÉS OXIDATIVO..

Tabla 2

Estado bioquímico, estrés oxidativo y metabolismo del hierro

\begin{tabular}{|c|c|c|c|}
\hline & Pacientes con EP idiopática & Controles & \\
\hline \multicolumn{4}{|c|}{ Parámetros bioquímicos de status nutricional. Media (SD) } \\
\hline Glucosa $(\mathrm{mg} / \mathrm{ml})$ & $103,94(22,93)$ & $112,37(37,86)$ & \\
\hline Urea $(\mathrm{mg} / \mathrm{ml})$ & $41,90(10,20)$ & $41,08(12,13)$ & \\
\hline Urato $(\mathrm{mg} / \mathrm{ml})$ & $4,96(1,16)$ & $5,39(1,13)$ & p 0,05 \\
\hline Colesterol total $(\mathrm{mg} / \mathrm{ml})$ & $216,81(38,51)$ & $215,77(38,25)$ & \\
\hline Triglicéridos $(\mathrm{mg} / \mathrm{ml})$ & $107,26(48,79)$ & $102,37(42,78)$ & \\
\hline Proteínas totales $(\mathrm{g} / \mathrm{dl})$ & $7,16(0,40)$ & $7,17(0,44)$ & \\
\hline Albúmina $(\mathrm{g} / \mathrm{dl})$ & $4,48(0,22)$ & $4,43(0,26)$ & \\
\hline \multicolumn{4}{|c|}{$\begin{array}{l}\text { Niveles plasmáticos de diversas moléculas relacionadas con } \\
\text { el estrés oxidativo }\end{array}$} \\
\hline $\mathrm{GSH}(\mathrm{mol} / \mathrm{ml})$ & $0,10(0,06)$ & $0,29(0,12)$ & p 0,001 \\
\hline MDA $(\mathrm{nmol} / \mathrm{ml})$ & $15,46(7,99)$ & $16,96(4,09)$ & \\
\hline Vitamina $\mathrm{E}(\mathrm{g} / \mathrm{ml})$ & $11,41(2,61)$ & $12,44(3,88)$ & \\
\hline Betacaroteno $(\mathrm{g} / \mathrm{ml})$ & $0,07(0,05)$ & $0,07(0,06)$ & \\
\hline Licopeno $(\mathrm{g} / \mathrm{ml})$ & $0,11(0,09)$ & $0,14(0,13)$ & \\
\hline \multicolumn{4}{|c|}{$\begin{array}{l}\text { Niveles plasmáticos de diversas moléculas relacionadas con } \\
\text { el metabolismo del hierro }\end{array}$} \\
\hline Hierro (ug/dl) & $91,21(35,05)$ & $87,18(29,72)$ & \\
\hline Ferritina (ug/l) & $148,70(191,10)$ & $97,66(98,20)$ & p 0,005 \\
\hline Transferrina (mg/dl) & $330,59(52,70)$ & $264,05(47,89)$ & p 0,001 \\
\hline Índice de sat. Transferrina (\%) & $27,53(11,35)$ & $32,91(13,52)$ & p 0,05 \\
\hline
\end{tabular}

Tabla 3

Odds Ratio bruta y ajustada * por ingesta calórica, por quartiles, para el consumo de diversos nutrientes

\begin{tabular}{|lccccccc|}
\hline & $Q$ & N1 Casos & N2 Controles & OR cruda & $p$ & OR ajustada * \\
\hline Vitamina C & 1 & 15 & 35 & 1 & - & 1 & - \\
& 2 & 16 & 33 & $1,13(0,48-2,65)$ & N.S. & $0,73(0,43-1,23)$ & N.S. \\
& 3 & 27 & 23 & $2,74(1,20-6,23)$ & 0,04 & $1,90(1,14-3,22)$ & 0,01 \\
& 4 & 23 & 26 & $2,06(0,9-4,71)$ & N.S. & $1,36(0,82-2,29)$ & N.S. \\
\hline Vitamina E & 1 & 27 & 22 & 1 & - & 1 & $0,81(0,48-1,35)$ \\
& 2 & 17 & 31 & $0,45(0,2-1,01)$ & & $1,05(0,64-1,76)$ \\
& 3 & 20 & 30 & $0,54(0,24-1,21)$ & & $0,82(0,48-1,36)$ \\
\hline
\end{tabular}

$\mathrm{Q}=$ cuartiles; $\mathrm{N} 1=\mathrm{n} .{ }^{\circ}$ de casos; $\mathrm{N} 2=\mathrm{n} .{ }^{\circ}$ de controles.

Entre paréntesis: intervalo de confianza al $95 \%$

Se han encontrado diferencias significativas $(p<0,001)$ en los niveles plasmáticos de GSH entre casos $(0,10 \mathrm{~mol} / \mathrm{ml} \quad 0,06)$ y controles $(0,29 \mathrm{~mol} / \mathrm{ml} \quad 0,12)$, siendo los niveles claramente menores en los casos. Los niveles de MDA, vitamina E, licopeno $\mathrm{y}$ betacarotenos, no diferían entre casos y controles (tabla 2). 
Al comparar los niveles plasmáticos de estas sustancias entre pacientes tratados con L-Dopa y no tratados, se encontraron diferencias significativas para los niveles de betacarotenos entre ambos grupos, pero no para el resto de parámetros (tabla 4).

En el grupo de pacientes con EP $(n=79)$, los niveles de GSH se correlacionan con la edad ( $\mathrm{r}=0,42 ; \mathrm{p}$ 0,001), de manera que los niveles son mayores a medida que ésta aumenta. Al analizar esta correlación por sexos se pone de manifiesto que la correlación existe en los hombres $(n=45 ; r=0,47$; $\mathrm{p} 0,05)$ pero no en las mujeres $(n=24$; $r=0,27 ; p=0,2)$. En el grupo control no se encuentra esta correlación con la edad. Tampoco existe correlación con el tiempo de evolución de los síntomas ni con las puntuaciones en la escala UPDRS.

Respecto al metabolismo del hierro, y aunque siempre dentro de los rangos considerados normales, en los pacientes con EP se encontraron valores significativamente mayores de ferritina ( $\mathrm{p} 0,05)$ y transferrina ( $\mathrm{p} 0,01$ ) que en los controles, con un índice de saturación de la transferrina menor (p 0,05). El hierro no mostró cambios significativos entre casos y controles (tabla 2).

El tratamiento con L-Dopa no modificó los niveles séricos de los parámetros del metabolismo del hierro estudiados. No existe correlación entre los niveles séricos de hierro y los parámetros antioxidantes, ni con el tiempo de evolución de la enfermedad.

\section{DISCUSIÓN}

La similitud de los datos referentes a la distribución demográfica y estado nutricional, garantiza la comparabilidad de los dos grupos estudiados (tablas 1 y 2). La constatación de que tanto los casos como los controles presentan niveles de glúcidos, lípidos y uratos dentro del rango de la normalidad, nos pareció especialmente importante, teniendo en cuenta que tanto la hiperuricemia como la hiperlipidemia podían alterar las determinaciones de GSH y MDA respectivamente. La diferencia encontrada en el nivel de ácido úrico entre casos y controles (mayores en este último grupo), podría estar en relación con la propia enfermedad. En este sentido diversos autores han publicado modificaciones en los niveles de ácido úrico en la EP, encontrándose niveles menores en cerebro y plasma ${ }^{17,31,32}$. Este hecho estaría en consonancia con el efecto antioxidante del ácido úrico y su posible papel protector frente a la EP. En nuestro estudio, establecimos como factor de exclusión la hiperuricemia (ácido úrico $>7,5$ ), y de este modo, de una muestra inicial seleccionada de 82 casos y 131 controles, eliminamos del análisis a 3 casos y a 24 controles. Esto significa que un $3,65 \%$ de los casos presentaba hiperuricemia constatada bioquímicamente, frente a un $18,32 \%$ de los controles. Este dato viene a apoyar el hallazgo de que en los pacientes con EP existe un menor nivel de urato que en la población control de la misma edad, a la vez que una menor incidencia de hiperuricémicos.

Tabla 4

Efecto del tratamiento con L-Dopa en los niveles plasmáticos de diversas sustancias

\begin{tabular}{|lcrl|}
\hline & Pacientes tratados & Pacientes no tratados & $p$ \\
\hline $\mathrm{GSH}(\mathrm{mol} / \mathrm{ml})$ & $0,10(0,06)$ & $0,11(0,06)$ \\
$\mathrm{MDA}(\mathrm{nmol} / \mathrm{ml})$ & $15,96(8,29)$ & $12,91(5,85)$ & \\
Vitamina E $(\mathrm{g} / \mathrm{ml})$ & $11,26(2,55)$ & $12,15(2,98)$ & \\
Betacaroteno $(\mathrm{g} / \mathrm{ml})$ & $0,06(0,04)$ & $0,12(0,09)$ & $\mathrm{p}<0,05$ \\
Licopeno $(\mathrm{g} / \mathrm{ml})$ & $0,10(0,08)$ & $0,13(0,13)$ & \\
\hline
\end{tabular}


La comprobación de un adecuado estado nutricional para ambos grupos, aporta validez a las diferencias que puedan encontrarse en el hábito de consumo de antioxidantes. En este sentido, no se encontraron diferencias significativas en el consumo estimado de vitamina $\mathrm{C}$ ni vitamina $\mathrm{E}$ entre ambos grupos, una vez ajustados los datos a la ingesta calórica (tabla 3). La ausencia de diferencias en los niveles plasmáticos de vitamina E, -carotenos y licopeno, entre casos y controles, está en concordancia con los hallazgos de consumo estimado. Dedicamos especial esfuerzo en la determinación de licopeno, carotenoide de color rojo intenso que se encuentra en algunas frutas y verduras y al que recientes estudios epidemiológicos atribuyen una importante capacidad para prevenir el desarrollo de enfermedades degenerativas y algunos tumores ${ }^{33}$.

Morens y cols ${ }^{19}$ demostraron una disminución de la ingesta de vitamina $\mathrm{E}$ en un grupo de pacientes con EP, aunque estudios posteriores sobre dieta y EP concluyen que sólo la ingesta de grasa animal y alimentos ricos en vitamina $\mathrm{D}$ incrementaban el riesgo de padecer EP, y no la ingesta de vitaminas $\mathrm{A}, \mathrm{C}$ y E $\mathrm{E}^{34}$. Aunque a las vitaminas $\mathrm{C}$ y E se les ha atribuido un importante papel en la regulación de la muerte celular programada (apoptosis), recientes estudios realizados sobre neuronas in vitro demuestran la capacidad protectora de la vitamina $\mathrm{C}$ pero no de la vitamina $\mathrm{E}^{35}$. El estudio DATATOP ${ }^{36}$ realizado en pacientes parkinsonianos de novo, ha puesto de manifiesto que el tratamiento diario con dosis altas de vitamina E no supone ningún beneficio clínico. Sin embargo otros trabajos han señalado un efecto beneficioso de la vitamina $\mathrm{E}$ en retrasar la evolución de la enfermedad ${ }^{37}$. No se han encontrado tampoco disminuciones de los niveles de vitamina $\mathrm{E}$ en la sustancia negra ${ }^{38}$. En plasma, los niveles de esta vitamina han sido normales ${ }^{16,39}$ o más bajos.

En nuestro estudio no hemos encontrado diferencias en el consumo de vitaminas $\mathrm{C} \mathrm{y}$ $\mathrm{E}$, por lo que consideramos que su ingesta como componentes de la dieta normal, no debe ser determinante en el desarrollo de la enfermedad.

Hemos encontrado niveles plasmáticos más bajos de GSH en los pacientes con EP que en los controles. Este hallazgo está en consonancia con lo que se observa en la sustancia negra de cerebros de pacientes con EP: una marcada reducción de GSH, que no se presenta en ninguna otra de las enfermedades neurodegenerativas de los ganglios basales. El glutatión es un tripéptido sintetizado por la propia célula con un paso limitante en la síntesis de la cisteína por la enzima cistationasa, y que podría alterarse por el envejecimiento $^{40}$. Esto abocaría a la célula a una situación de desprotección frentre a la acción lesiva de los radicales libres, facilitando el desarrollo de procesos degenerativos. Tal es el caso de la sustancia negra, donde la síntesis y metabolización de dopamina genera formas oxidadas de la misma o metabolitos como la neuromelanina (polímero de la dopamina oxidada), que acumulan hierro, facilitando el estrés oxidativo ${ }^{35}$. El GSH parece que ejerce un papel protector fundamental, regulando incluso la activación de la apoptosis celular ${ }^{41}$. Trabajos sobre cultivos neuronales in vitro demuestran cómo la neuromelanina es capaz de inducir apoptosis celular, que puede prevenirse aportando al medio de cultivo GSH, N-acetilcisteína (que aporta cisteína a la vía sintética del glutatión sobrepasando la enzima cistationasa) o vitamina $\mathrm{C}^{35}$. Un ensayo terapéutico con GSH intravenoso en pacientes con EP de reciente instauración, demostró una mejoría de los trastornos motores de hasta un $42 \%$ en el grupo sometido a tratamiento, y este efecto se prolongó hasta 2 meses después de finalizado el mismo ${ }^{42}$. Esto hace presumir, que unos niveles más elevados en sangre periférica de GSH mejorarían la evolución de la enfermedad.

La correlación positiva entre los niveles de GSH y la edad en los casos, encontrada en nuestro trabajo, no es fácil de interpretar. Según nuestros resultados, los pacientes de 
más edad tienen niveles de GSH mayores que los pacientes más jóvenes. Pero esta correlación parece ser a expensas de los hombres, puesto que al analizar separadamente los niveles de GSH en hombres y en mujeres se aprecia cómo la correlación es sólo significativa en los hombres ( $r=0,47 ; \mathrm{p} 0,05)$. Esto nos induce a pensar que los pacientes varones más jóvenes estarían sometidos a mayor estrés oxidativo, quizás porque en ellos incidan factores de exposición prooxidantes con mayor peso que en pacientes de más edad o en las mujeres. En este sentido, en un estudio previo publicado por nuestro grupo $^{43}$, encontramos que el perfil epidemiológico de la EP idiopática es diferente en los hombres que en las mujeres, con una mayor incidencia y mayor precocidad de aparición en los hombres. Ambos hallazgos apuntan a que los factores de exposición en la EP no actúan de modo semejante en hombres y mujeres.

No hemos hallado diferencias significativas en los niveles plasmáticos de GSH entre pacientes tratados $y$ no tratados con L-Dopa. Tohgi et $\mathrm{al}^{8}$, determinaron los niveles de glutatión en líquido cefalorraquídeo, no encontrando diferencias entre pacientes con EP y controles para la forma reducida (GSH), pero sí para la forma oxidada (GSSG). En su estudio, el tratamiento con L-Dopa elevó los niveles de GSH en LCR. Spencer et $\mathrm{al}^{44}$ también encuentran una disminución de GSH en cerebros de pacientes con EP en relación con la formación de conjugados entre el GSH y derivados de la oxidación de L-Dopa. Los cambios encontrados en LCR podrían ser compensatorios a la presencia de formas oxidadas de L-Dopa, aspecto que ha inducido a pensar que el tratamiento con L-Dopa alteraría los niveles plasmáticos de ciertos antioxidantes. En nuestro estudio los niveles de GSH son similares en pacientes tratados y no tratados.

Los niveles de MDA en plasma no mostraron diferencias entre pacientes y controles. Otros grupos tampoco han encontrado cambios en el MDA ${ }^{16,17}$. Sí se ha encontrado elevado en sustancia negra ${ }^{5}$, como expresión del aumento de la peroxidación lipídica. El no encontrarse aumentado en plasma probablemente se deba a la escasa especificidad de la molécula, ya que en la economía humana se producen múltiples fenómenos oxidativos que generan MDA.

Aunque dentro de los límites de la normalidad, hemos encontrado unos niveles plasmáticos de transferrina y ferritina mayores en los pacientes con EP que en los controles, mientras que el índice de saturación de la transferrina está disminuido. Este hecho podría representar una avidez incrementada para la captación, transporte y depósito tisular de hierro en los pacientes con EP. Este hallazgo difiere de los datos recogidos en otros trabajos ${ }^{10,45}$, en los que se encuentran niveles plasmáticos menores de ferritina, transferrina, su índice de saturación y el hierro libre. En la sustancia negra sin embargo, todos los grupos encuentran incremento del hierro y una disminución de la ferritina ${ }^{46,47}$, interpretándose esto último como un fallo en los mecanismos de buffer del hierro. En la hipótesis patogénica del estrés oxidativo en el desarrollo de la EP, el hierro jugaría un papel muy importante, ya que él mismo es capaz de catalizar la formación de radicales hidroxilo ${ }^{41}$ y con ello reacciones oxidativas no enzimáticas. Además, el hierro libre facilita la activación de la vía de la ciclooxigenasa, activando la fosfolipasa A2 y junto a factores de agregación plaquetar induce adhesión leucocitaria que aporta al medio grandes cantidades de $\mathrm{H} 2 \mathrm{O} 2^{48}$. Las modificaciones en el metabolismo del hierro plasmático encontradas en nuestro trabajo, están en consonancia con lo que ocurre a nivel de la sustancia negra, por lo que creemos que este hallazgo debe ser tenido en cuenta en trabajos que investiguen la fisiopatogenia de la enfermedad.

\section{AGRADECIMIENTOS}

Al Departamento de Salud, por la financiación del proyecto y al Servicio Navarro 
de Salud, por el apoyo logístico al proyecto a través del Centro de Investigación Biomédica y del Centro de Especialidades Príncipe de Viana. A los Neurólogos del Sistema Sanitario de Navarra que remitieron los enfermos al estudio: T. Ayuso, M. Bujanda, G. Delgado, J. Gállego, F. Jarauta, F. Lacruz, JM. Manubens, E. Maraví, F. Martínez Ibáñez, P. Quesada, G. Soriano y JA. Villanueva. A M. ${ }^{a}$ José Lasheras, Diplomada universitaria en dietética y Diplomada universitaria en enfermería. A Aurelio Barricarte, director en Navarra del Proyecto europeo dieta y cáncer (EPIC) y a Josep Soldevilla, Director de Ndiet Soft S.L. por las correspondientes autorizaciones para la utilización del programa informático EPIC-Soft, y a Carlos Alberto González por la explotación informática de la historia de dieta. Al Laboratorio del Centro de Especialidades Príncipe de Viana. Servicio Navarro de Salud. A Celina Equiza y Maribel Vidaurre por su asistencia.

\section{BIBLIOGRAFÍA}

1. Sian J, Dexter DT, Lees AJ, Daniel S, Agid Yet al. Alterations in glutathione levels in Parkinson's disease and other neurodegenerative disorders affecting basal ganglia. Ann Neurol 1994; 36: $348-355$.

2. Pearce RKB, Owen A, Daniel S, Jenner P, Marsden CD. Alterations in the distribution of glutathione in the substantia nigra in Parkinson's disease. J Neural Transm 1997; 104: 661-667.

3. Ferrer JV, Sastre J., Pallardó FV, Asensi M., Viña J. and Miquel J. Senile Cataract: A Review on Free Radicals Patogénesis and Antioxidant Prevention. Arch. Gerontol Geriatric 1991; 13:51-59.

4. Jenner P. Oxidative stress as a cause of Parkinson's disease. Acta Neurol Scand (Supl 1) 1991; 136: 6-15.

5. Schapira AHV. Evidence for mitochondrial dysfunction in Parkinson's disease: a critical appraisal. Mov Disord 1994; 9: 125-138.

6. García de la Asunción J, Millán A, Pla R, Bruseghini L, Esteras A, Pallardó FV et al. Mitochondrial glutathione oxidation correlates with age-associated damage to mitochondrial DNA. FASEB 1996; 10: 333-8.
7. Dexter DT, Carayon A, Javoy-Agid F, Agid Y, Wells FR, Daniel SE et al. Alterations in the levels of iron, ferritine and other tracer metals in Parkinson's disease and other neurodegenerative diseases affecting the basal ganglia. Brain 1991; 114: 1953-1975.

8. Tohgi H, Abe T, Saheki M, Hamato F, Sasaki K, Takahashi S. Reduced and oxidized forms of glutathione and alpha-tocopherol in the cerebrospinal fluid of parkinsonian patients: comparison between before and after 1-Dopa treatment. Neurosci Lett 1995; 184: 21-24.

9. Molina JA, de Bustos F, Jiménez-Jiménez FJ et al. Cerebrospinal fluid levels of alpha-tocopherol (vitamin E) in Parkinson's disease. J Neural Transm 1997; 104: 1287-1293.

10. Logroscino G, Marder K, Graziano J et al. Altered systemic iron metabolism in Parkinson's disease. Neurology 1997; 49: 714-717.

11. Logroscino G, Marder K, Graziano J et al. Dietary iron, animal fats, and risk of Parkinson's disease. Mov Disord 1998; 13 (Suppl 1): 13-16.

12. Molina JA, Jiménez-Jiménez FJ, Gómez $\mathrm{P}$ et al. Decreased cerebrospinal fluid levels of neutral and basic amino acids in patients with Parkinson's disease. J Neurol Sci 1997; 150: 123-127.

13. Jiménez-Jiménez FJ, Fernández-Calle, Molina JA. Serum levels of vitamin A in Parkinson's disease. J Neurol Sci 1992; 111: 73-76.

14. Jiménez-Jiménez FJ, Fernández-Calle, Molina JA. Serum levels of b-carotene and others carotenoids in Parkinson's disease. Neurosci Lett 1993; 157: $103-106$

15. Davis JW, Grandinetti A, Waslien CI, Ross GW, White LR, Morens DM. Observations on serum uric acid levels and the risk of idiopathic Parkinson's disease. Am J Epidemiol 1996; 144 (5): 480-484.

16. Ahlskog EJ, Vitti RJ, Low PA, Tyce JM. No evidence for sistemyc stress in Parkinson's or Alzheimer's disease. Mov Disord 1995; 19: 566-573.

17. González-Fraguela ME, Céspedes E, Arencibia $\mathrm{R}$, Broche $\mathrm{F}$, Gómez A. Indicadores de estrés oxidativo y efecto del tratamiento antioxidante en pacientes con enfermedad de Parkinson primaria. Rev Neurol 1998; 26: 28-33.

18. Golbe LI, Farrell TLM, Davis PH. Case-control study of early life dietary factors in Parkinson's disease. Arch Neurol 1988; 45: 1350-1353. 
19. Morens DM, Grandinetti A, Waslien CI, Park CB, Ross GW, White LR. Case-control study of idiopathic Parkinson's disease and dietary vitamin E intake. Neurology 1996; 46: 1270-1274.

20. Gorell JM, Johnson CC, Rybicki BA, Booker P, Wulbrecht N, Sanders K. Nutrient intake in Parkinson's disease. Neurology 1989; 39: 181 .

21. Scheider WL, Hershey LA, Vena JE et al. Dietary antioxidants and other dietary factors in the etiology of Parkinson's disease. Mov Disord 1997; 12: $190-196$

22. Hughes AJ, Daniel SE, Kilford L, Lees AJ. Accuracy of clinical diagnosis of idiopathic Parkinson's disease: a clinico-pathological study of 100 cases. J Neurol Neurosurg Psychiatry 1992; 55: 181-184.

23. Stern MB. The clinical characteristics of Parkinson's disease and Parkinsonian syndrome: diagnosis and assessment. En: Stern MB, Hurtig HI, editores. The comprehensive management of Parkinson's disease. Nueva York: PMA Publishing Company 1988; 3-50.

24. Hoehn MM, Yahr MD. Parkinson: onset, progression and mortality. Neurology 1967; 17: 427-442.

25. Larumbe R, Viñes JJ, Guillén F, Gaminde I, Artázcoz MT. Asociación inversa entre consumo de tabaco y enfermedad de Parkinson idiopática. Anales Sis San Navarra 1999; 22 (1): 17-23.

26. Folstein MJ, Folstein SE, Mc Hug PR Mini-Mental State. A practical grading for cognitive state of patients for the clinical. J Psychiatry Res 1975; 12: 189-198.

27. Grupo EPIC de España. El estudio prospectivo europeo sobre dieta, cáncer y salud (EPIC) en España. Med Clin (Barc) 1994; 102: 781-785.

28. Brigelius R, Muckel C, Akerboom TPM, Sies H. Biochem Pharmacol 1983; 32: 2592-2534.

29. Thurnham DI, Smith S, Flora PS. Concurrent Liquid-Chromatographic Assay of Retinol, a-tocoferol, b-Carotene, Lycopene, and b-Cryptoxanthin in Plasma, with Tocopherol Acetate as Internal Standard. Clin Chem 1988; 34: 377-381.

30. Buege JA, Aust SD. Method of Enzimology 1973; 52: 302.

31. Church WH, Ward VL. Uric acid is reduced in the substantia nigra in Parkinson's disease: effect on dopamine oxidation. Brain Res Bull 1994; 33 : 419-425.
32. Tohgi H, Abe T, Takahashi S, Kikuchi T. The urate and xanthine concentrations in the cerebrospinal flid in patients with vascular dementia of the Binswanger type, Alzheimer type dementia, and Parkinson's disease. J Neural Transm Park Dis Dement Sect 1993; 6: 119-126.

33. Astorg P, Gradelet S, Berges R, Suschetet M. Dietary lycopene decreased the initiation of liver preneoplasic foci by diethylnitrosamine. Nutr Cancer 1997; 29: 60-68.

34. Anderson C, Checkoway H, Franklin GM, Beresford S, Smith-Weller T, Swanson PD. Dietary factors in Parkinson's disease: the role of food groups and specific foods. Mov Disord 1999; 14: $21-7$

35. Offen D, Ziv I, Sternin H, Melamed E, Hochman A. Prevention of dopamine-induced cell death by thiol antioxidants: possible implications for treatment of Parkinson's disease. Exp Neurol 1999; 141: 32-9.

36. Shoulson I. DATATOP: A decade of neuroprotective inquiry. Parkinson Study Group. Deprenyl and Tocopherol Antioxidative Therapy of Parkinsonism. Ann Neurol 1998; 44 (supl 1): S160-166.

37. Fahn S. A pilot trial of high-dose alpha-tocopherol and ascorbate in early Parkinson's disease. Ann Neurol 1992; 32 (Supl): 128-132.

38. Dexter DT, Ward RH, Wells FR, Daniels SE, Lees AJ, Peters TJ et al. -tocopherol levels in brain are not altered in Parkinson's disease. Ann Neurol 1992; 32: 591-593

39. Fernández-Calle $\mathrm{P}$, Molina JA, Jiménez-Jimenez FJ, Vazquez A, Pondal M, García-Ruiz P et al. Serum levels of alpha-tocopherol (vitamin E) in Parkinson's disease. Neurology 1992; 42: 1064-1066.

40. Ferrer J.V., Gascó E., Sastre J., Pallardó F.V., Asensi M., and Viña J. «Age Related Changes in Glutathione Syntesis in the Age Lens». Biochemical J. 1990; 269: 531-34.

41. 41.Hirsch EC, Faucheux BA. Iron metabolism and Parkinson's disease. Mov Disord 1998; 13: $39-45$.

42. Sechi G, Deledda MG, Bua G, Satta WM, Deiana GA, Pes GM, Rosati G. Reduced intravenous glutathione in the treatment of early Parkinson's disease. Prog Neuropsychopharmacol Biol Psychiatry 1996; 20: 1159-1170.

Rev Esp Salud Pública 2001, Vol. 75, N. ${ }^{\circ} 1$ 
ESTUDIO CASO-CONTROL DE MARCADORES DE ESTRÉS OXIDATIVO..

43. Viñes JJ, Larumbe R, Gaminde I, Artázcoz MT Incidencia de la enfermedad de Parkinson idiopática y secundaria en Navarra. Registro poblacional de casos. Neurología 1999; 14: 16-22.

44. Spencer JP, Jenner P, Daniel SE, Lees AJ, Marsden DC, Halliwell B. Conjugates of catecholamines with cysteine and GSH in Parkinson's disease: possible mechanisms of formation involving reactive oxygen species. J Neurochem 1998; 71: 2112-2122.

45. Abbott RA, Cox M, Marcus H,Tomkins A. Diet, body size and micronutrient status in Parkinson's disease. Eur J Clin Nutr 1992; 46: 879-884.
46. Connor JR, Snyder BS, Arosio P, Loffler DA, LeWitt P. A quantitative analysis of isoferritins in select regions of aged, parkinsonian, and Alzheimer's diseased brains. J Neurochem 1995; 65: 717-724.

47. Fahn S, Cohen G. The oxidant hypothesis in Parkinson's disease: evidence supporting it. Ann Neurol 1992; 32: 804-12.

48. Ferrer JV, Herrera J, Balén E, Lera JM. Utilización de órganos procedentes de donantes en parada cardíaca: Revisión de la patogenia del daño orgánico y los posibles mecanismos de prevención. Anales Sis San Navarra 1997; 20, 1: 47-56. 
